\title{
Is "How Are You Doing" Enough? Need for Tailored Questions to the Patients With Functional Dyspepsia
}

\author{
Jaehoon Jahng and Yong Sung Kim* \\ Division of Gastroenterology, Department of Internal Medicine, Digestive Disease Research Institute, Wonkwang University Sanbon Hospital, \\ Gunpo, Gyeonggi-do, Korea
}

Article: Impact of eating attitude and impairment of physical quality of life between tertiary clinic and primary clinic functional dyspepsia outpatients in Japan

Shimpuku M, Futagami S, Tajima N, et al

(J Neurogastroenterol Motil 2014;20:506-515)

For some time, we have known that functional dyspepsia (FD) or other functional gastrointestinal (GI) disorders are associated with non-organic causes such as sleep disorders, eating habits and levels of anxiety, whether they maybe the cause or the end result. ${ }^{1-3}$ However, we tend to think that FD patients visiting tertiary clinic may present with severe degree of symptoms, psychological problems and impaired quality of life than those going to primary clinic.

In this month's edition of JNM, Shimpuku et al ${ }^{4}$ have shown that this is not so at least in Japan. There was no significant difference in mean GI Symptom Rating Scale (GSRS) which represents various FD symptoms, or Pittsburg Sleep Quality Index (PSQI) scores which indicates sleep disorders between tertiary clinic and primary clinic FD outpatients. Interestingly, state of anxiety and trait of anxiety, which have been known as main determinants for health care seeking behavior, ${ }^{5-7}$ were also not different between tertiary and primary clinics, although they were higher than healthy volunteers. In this study, the presence of psychological stress does implicate on the pathophysiology of FD, however, the gravity of psychological comorbidities seem to have less effect on the decision to go to primary or tertiary clinics. ${ }^{4}$ Rather, they reported that impairment of physical quality of life measured by Social Function-8 (SF-8) test was more important factor for healthcare-seeking behavior than impairment of mental quality of life in FD patients visiting tertiary clinic compared to those visiting primary clinic. ${ }^{4}$

It is worthy to point out that above results about determinant factors for health care seeking behavior should be interpreted under consideration of different referral systems, ease of accessibility to healthcare facilities and diverse cultural factors in each country. In Domestic/International Gastroenterology Surveillance Study performed in 1999, Japanese subjects with relevant

Received: September 4, 2014 Revised: None Accepted: September 14, 2014

(c) This is an Open Access article distributed under the terms of the Creative Commons Attribution Non-Commercial License (http://creativecommons. org/licenses/by-nc/3.0) which permits unrestricted non-commercial use, distribution, and reproduction in any medium, provided the original work is properly cited.

${ }^{*}$ Correspondence: Yong Sung Kim, MD, PhD

Department of Gastroenterology, Wonkwang University Sanbon Hospital, 321, Sanbon-ro, Gunpo-si, Gyeonggi-do 435-804, Korea

Tel: +82-31-390-2975, Fax: +82-31-398-2223, E-mail: wms89@hanmail.net

Financial support: This work was supported by Wonkwang University, 2013.

Conflicts of interest: None.

Author contributions: Jaehoon Jahng, drafting manuscript; Yong Sung Kim, drafting manuscript, critical revision and final approval.

ORCID: Yong Sung Kim, http://orcid.org/0000-0001-8836-4818. 
upper GI symptoms were found to have a notably higher consultation rate than those from other western countries. ${ }^{8}$ Therefore, the characteristics of patients in tertiary hospital in each country were not the same among studies. This difference between countries is not only from the delay in referral system to meet a specialist, but also from the cost barrier and cultural differences. ${ }^{9,10}$

Shimpuku et $\mathrm{al}^{4}$ also showed that eating habits were different between FD patient and healthy control groups. Although, food is suspected to be closely associated with GI symptom generation in FD patients, only a few studies have been investigated, and the results were inconsistent. ${ }^{11}$ The type of food, type of nutrients, frequency of meals and volume of each meal may altogether affect GI symptoms. ${ }^{11}$ In this study, Shimpuku et $\mathrm{al}^{4}$ showed that taking dinner at night was not related to FD, but the frequency of eating between meals was higher in primary clinic patients compared to that in tertiary clinic FD patients. This result is somewhat different compared to previous western report, which showed FD patients eat fewer meals than healthy subjects, however the number of light meals or snacks was not different between FD and healthy subject. ${ }^{11}$ This maybe due to the tendency to avoid food at night or avoid taking large meal out of fear of developing abdominal discomfort. ${ }^{11}$ So, primary care patients in this study might already have known how to reduce their symptoms by eating small meals frequently. For meal pattern in FD patients, the characteristics and volume of typical meal of each country should also be considered. For example, large fatty meal in western countries would surely create more indigestion and discomfort compared to a relatively smaller in volume, less fatty meal in Asian countries.

However, the role of fatty meal in symptom severity in FD patient was reconfirmed in this study and it showed positive correlation to PSQI scores. ${ }^{4,11,12}$ Shimpuku et $\mathrm{al}^{4}$ reported that higher fat consumption was more related to FD patients visiting tertiary clinic compared to those visiting primary clinic. In addition, the association of total GSRS scores with global PSQI scores in both tertiary and primary clinic FD patients tell us that sleep disorders and GI disturbances are closely related in Asian populations, too. But, whether GI symptoms lead to sleep disorders or sleep disorders contribute to induce GI symptoms is unclear for the moment and left for future investigations.

So, would it be enough just to ask the patient visiting primary or tertiary clinic with FD if his or her GI symptom is okay? The answer is no. Physicians should consider that the patient suffers not only from their GI symptoms, but also from decreased quality of life by disturbed eating patterns and non-GI symptoms such as sleep disturbance. And these factors should be tailored according to available health care system and cultural differences. More and more, we begin to understand that there are subtle but distinctive differences of clinical symptoms and associated factors between FD and other functional GI disorders. And we need to be prepared to ask these subtle differences to better treat patients with FD whether they are visiting primary clinic or tertiary clinic.

\section{References}

1. Talley NJ, Zinsmeister AR, Schleck CD, Melton LJ 3rd. Dyspepsia and dyspepsia subgroups: a population-based study. Gastroenterology 1992;102(4 Pt 1):1259-1268.

2. Castillo EJ, Camilleri M, Locke GR, et al. A community-based, controlled study of the epidemiology and pathophysiology of dyspepsia. Clin Gastroenterol Hepatol 2004;2:985-996.

3. Ford AC, Forman D, Bailey AG, Cook MB, Axon AT, Moayyedi P. Who consults with dyspepsia? Results from a longitudinal 10-yr follow-up study. Am J Gastroenterol 2007;102:957-965.

4. Shimpuku M, Futagami S, Tajima N, et al. Impact of eating attitude and impairment of physical quality of life between tertiary clinic and primary clinic functional dyspepsia outpatients in Japan. J Neurogastroenterol Motil 2014;20:506-515.

5. Koloski NA, Talley NJ, Boyce PM. Epidemiology and health care seeking in the functional GI disorders: a population-based study. Am J Gastroenterol 2002;97:2290-2299.

6. Cheng C. Seeking medical consultation: perceptual and behavioral characteristics distinguishing consulters and nonconsulters with functional dyspepsia. Psychosom Med 2000;62:844-852.

7. $\mathrm{Hu} \mathrm{WH}$, Wong WM, Lam CL, et al. Anxiety but not depression determines healthcare-seeking behavior in Chines patients with dyspepsia and irritable bowel syndrome: a population-based study. Aliment Pharmacol Ther 2002;16:2081-2088.

8. Haycox A, Einarson T, Eggleston A. The health economic impact of upper gastrointestinal symptoms in the general population: results from the Domestic/International Gastroenterology Surveillance Study (DIGEST). Scand J Gastroenterol 1999;231(suppl):38-47.

9. David S, Robin O, Sarah T, Miraya J. International profiles of health care systems, 2013: Australia, Canada, Denmark, England, France, Germany, Italy, Japan, the Netherlands, New Zealand, Norway, Sweden, Switzerland, and the United States. Available URL: http:// www.commonwealthfund.org/publications/fund-reports/2013/nov/ international-profiles-of-health-care-systems (accessed 1 September 2014).

10. Saito AI. A Japanese physician's experience as an inpatient in the United States and Japan. Arch Gynecol Obstet 2011;283(suppl 1):105-106.

11. Pilichiewicz AN, Horowitz M, Holtmann GJ, Talley NJ, FeinleBisset C. Relationship between symptoms and dietary patterns in patients with functional dyspepsia. Clin Gastroenterol Hepatol 2009;7: 317-322.

12. Carvalho RV, Lorena SL, Almeida JR, Mesquita MA. Food intolerance, diet composition, and eating patterns in functional dyspepsia patients. Dig Dis Sci 2010;5 5:60-65. 